\title{
Incidencia y patrones de transmisión de rubeola en México
}

\author{
José Luis Díaz-Ortega, MC, (I) Carlos D. Meneses-Reyes, MC, (2) \\ Manuel Palacios-Martínez, MC, M en C. ${ }^{(1)}$
}

\begin{abstract}
Díaz-Ortega JL, Meneses-Reyes CD, Palacios-Martínez M. Incidencia y patrones de transmisión de rubeola en México. Salud Publica Mex 2007;49:337-344.
\end{abstract}

\section{Resumen}

Objetivo. Describir los cambios en los patrones de transmisión de rubeola en México. Material y métodos. Se analizó la incidencia durante el periodo 1990 a 2005 y se calcularon la tendencia por regresión lineal simple y el corredor endémico por transformación logarítmica de la media geométrica de las tasas de incidencia mensual y recorridos superior e inferior por IC95\%. Resultados. La morbilidad mostró una tendencia estable de 1990 a 1998 y descendente de 1999 a $2005\left(r=-0.88, r^{2}=0.77\right)$, con disminución acumulada de $97.1 \%$. Además, se observaron la atenuación de la variación estacional, la interrupción transitoria de transmisión en 13 estados y el aumento de la proporción de casos en personas de 15 a 44 años y menores de un año, después del inicio de la vacunación antes de los siete años y de la estrategia combinada de inmunización (niños y adultos), respectivamente, con reducción de la incidencia en ambos grupos. Conclusiones. El reforzamiento de la vigilancia y la vacunación sistemática y suplementaria favorecerá la interrupción endémica y la propuesta de su eliminación continental para 2010.

Palabras clave: rubeola; epidemiología; México
Díaz-Ortega JL, Meneses-Reyes CD, Palacios-Martínez M. Incidence and transmission patterns of rubella in Mexico. Salud Publica Mex 2007;49:337-344.

\section{Abstraet}

Objective. To describe changes in the transmission patterns of rubella in Mexico. Material and Methods. An analysis of rubella incidence during 1990-2005 was performed to estimate the morbidity trend through a simple linear regression model. Endemic mapping was carried out by logarithmic transformation of the geometric mean of monthly incidence rates and $95 \% \mathrm{Cl}$ to estimate high and low endemic ranges. Results. A stable trend was observed in morbidity during 1990-1998, descending during 1999-2005 $\left(r=-0.88, r^{2}=0.77\right)$, with a $97.1 \%$ cumulative decrease.Attenuation during seasonal variation, temporary interruption of transmission in 13 states and increase in the proportion of cases in 15-44 year-olds as well as in children less than one year of age, have been observed respectively after starting vaccination of children less than seven years of age and the implementation of combined strategy (children and adults) for rubella immunization, with incidence decrease in both groups. Conclusions. Reinforcement of surveillance and routine-supplemental immunization activities would favor the interruption of endemic rubella and contribute to the continent-wide elimination proposal for 2010.

Key words: rubella; epidemiology; Mexico

(I) Centro de Investigación Sobre Enfermedades Infecciosas, Instituto Nacional de Salud Pública. Cuernavaca, México.

(2) Centro Nacional para la Salud de la Infancia y la Adolescencia, Secretaría de Salud. México.

Fecha de recibido: 8 de enero de 2007 - Fecha de aceptado: 13 de junio de 2007

Solicitud de sobretiros: Dr. José Luis Díaz-Ortega. Instituto Nacional de Salud Pública. Avenida Universidad 655 , Col. Santa María Ahuacatitlán, 62508 Cuernavaca, Morelos, México.

Correo electrónico: jdiaz@correo.insp.mx 
$\mathrm{L}^{\mathrm{a}}$ a rubeola es un padecimiento viral febril exantemático, casi siempre benigno, que puede ocasionar aborto, mortinato o efectos teratógenos graves hasta en $69 \%$ de los productos de la gestación, cuando se adquiere la infección en el primer trimestre del embarazo. Sin embargo, la frecuencia de las malformaciones que constituyen el síndrome de rubeola congénita (SRC) puede ser de $90 \%$ si la infección se presenta en las primeras 11 semanas de gestación. ${ }^{1,2}$ Se ha estimado que cada año se presentan en el mundo 100000 casos de SRC.,4 Entre 1962 y 1965, Estados Unidos sufrió una pandemia de rubeola que ocasionó, en 1964 y 1965, 20000 nacimientos con SRC y 11000 muertes fetales, lo que puso de manifiesto la importancia de prevenir este padecimiento. ${ }^{2,3,5}$

Aunque la rubeola se consideraba con anterioridad un trastorno "propio de la niñez", algunas encuestas serológicas internacionales muestran una elevada susceptibilidad en mujeres en edad fértil (MEF), ${ }^{2-4}$ una situación en particular grave en países que no han introducido la vacunación específica o cuyas coberturas de vacunación son insuficientes; esto favorece la acumulación de sujetos susceptibles y el desplazamiento de la edad promedio de infección a grupos de mayor edad, ${ }^{6-8}$ lo cual incrementa la probabilidad de riesgo de presentar el SRC.

Aunque la rubeola es una enfermedad de notificación obligatoria en México, la eficiencia del registro de casos ha presentado notorias variaciones. Antes de 1990 se observaba pobre notificación de casos a la Secretaría de Salud, debido a que de 1989 a 1990 se presentó una epidemia de sarampión con 89163 casos notificados y cerca de 7000 defunciones. Se reforzó la vigilancia y el informe de enfermedades exantemáticas que, como la rubeola, requerían diagnóstico diferencial respecto del sarampión. ${ }^{9}$ En 1993, México se unió al compromiso regional para la eliminación del sarampión en el continente americano. De esa manera, con la finalidad de introducir una mayor sensibilidad a la detección de casos, se estableció el sistema de vigilancia de enfermedades febriles exantemáticas, cuya definición de caso sospechoso por la similitud del cuadro clínico incluía los casos de rubeola y ello reforzó de forma indirecta la notificación de esta enfermedad..$^{10}$ En 2003, México determinó eliminar la rubeola y SRC en el continente americano, según la propuesta de la Organización Panamericana de la Salud, y estableció de modo oficial el sistema de vigilancia de sarampión-rubeola, a partir de la vigilancia de enfermedades febriles exantemáticas. ${ }^{11-13}$

La vacunación universal contra la rubeola en niños de 1 y 6 años de edad se inició en México en 1998 con la introducción de la vacuna combinada contra sarampión, rubeola y parotiditis (SRP) en el programa regular de vacunación. En 2000 se vacunó con enfoque de riesgo a trabajadores de la salud, magisterio, turismo y fuerzas armadas contra sarampión y rubeola mediante la vacuna combinada SR y se estableció la vacunación sistemática a hombres y mujeres adultos en unidades de salud. En 2004 se aplicó esta vacuna en campañas de inmunización a hombres y mujeres de 13 a 39 años. ${ }^{14-16} \mathrm{El}$ propósito de este trabajo es describir la tendencia y evaluar los cambios en los patrones de transmisión de la rubeola adquirida o posnatal.

\section{Material y métodos}

Se realizó un estudio ecológico de casos de rubeola en México de 1985 a 2005. Se consultó la información publicada o disponible en los portales electrónicos de la Dirección General de Epidemiología de la Secretaría de Salud ${ }^{9}$ y el Consejo Nacional de Vacunación. ${ }^{16}$ Se analizaron los indicadores de incidencia anual nacional y por entidad federativa, así como el número de casos por mes, para describir su evolución epidemiológica en espacio, persona y tiempo, incluida la variación estacional de 1990 a 2005. Para evaluar posibles desplazamientos etarios de la enfermedad, se condujo un análisis de la distribución de casos por grupo de edad y se computó la incidencia para cada franja etaria. La información disponible sobre SRC fue insuficiente para introducir este padecimiento en la modelación.

En virtud de los cambios operados en el sistema de vigilancia epidemiológica y en las actividades de control de las enfermedades febriles exantemáticas en el país, se consideró que la información disponible configuraba una serie interrumpida de $\operatorname{casos}^{17}$ ya que en el lapso analizado se efectuaron intervenciones en el sistema de vigilancia y el programa de vacunación que modificaron los procedimientos de captación de datos en cuando menos cinco periodos: a) etapa de subregistro elevado de casos (anterior a 1990); b) etapa de mejora continua de la notificación (desde 1990); c) inicio de la vacunación universal con dos dosis de vacuna SRP aplicada al año y seis años de edad en 1998; d) inmunización a adultos con la vacuna SR con enfoque de riesgo en 2000; y e) campaña de vacunación con SR a hombres y mujeres de 13 a 39 años en 2004. La cobertura de vacunación en niños de 1 a 4 años ha presentado una evolución estable de 1998 a 2005 con media de $98.3 \%$ y límites de variación de 97.8 a $98.5 \%$. La información sobre la cobertura de vacunación en el grupo de seis años (segunda dosis del esquema) y la alcanzada en las estrategias de vacunación a adultos no se encontró disponible en las fuentes consultadas. ${ }^{9,16}$

Se calcularon el número de casos evitados por el programa de vacunación y la disminución de casos respecto de la vacunación indirecta (sólo niños de 
1 y 6 años) y la reducción acumulada al considerar la incorporación de adultos (medida de vacunación combinada: indirecta y directa en adultos de ambos sexos) a las actividades regulares y suplementarias de inmunización. Debido a que la vacuna SRP se introdujo en el país en octubre de 1998 para evaluar la tendencia de la morbilidad, se comparó el periodo prevacunal de 1990 a 1998 con el vacunal de 1999 a 2005 a través de un modelo de regresión lineal simple, en el que se consideró como variable dependiente la incidencia anual y como independiente el año de observación. ${ }^{17-20}$

Para elaborar el corredor endémico del trastorno de 1993 a 2005, a partir del número de casos notificados por mes de 1989 a 2005, se diseñó una plantilla de cálculo en Microsoft Excel ${ }^{19}$ y se calcularon las tasas mensuales de incidencia; de esa manera se obtuvo la transformación logarítmica de su media geométrica de 1989 a 2005 como indicador del valor esperado de casos nuevos para el mes y año iésimos analizados en los cinco años previos. Los recorridos superior e inferior del corredor se computaron a partir de los valores transformados, las desviaciones estándar y los intervalos de confianza a 95\% de las medias mensuales de incidencia. Para obtener las unidades originales se calculó el antilogaritmo de los valores transformados. Los resultados se analizaron en el espacio cartesiano mediante el asistente de gráficos de Excel. Para analizar la evolución endemoepidémica de la enfermedad ${ }^{21}$ se dividió el espacio fase del gráfico en cuatro zonas de interés epidemiológico: a) zona epidémica (por arriba del recorrido superior); b) zona de alarma (entre la media y el recorrido superior); c) zona de seguridad (entre la media y el recorrido inferior); y d) zona de éxito (por abajo del recorrido inferior). ${ }^{19}$

\section{Resultados}

La figura 1 muestra picos de morbilidad de menor magnitud en los años anteriores a 1990; de 1990 a 1998 se observaron tres exacerbaciones epidémicas (1990, 1993 y 1998), con tendencia relativamente estable $(r=-0.32$, $\left.r^{2}=0.10\right)$. La introducción de la vacunación universal a niños en octubre de 1998 se acompañó a partir de 1999 de una disminución de los picos de incidencia, con franca tendencia descendente en el periodo vacunal de 1999 a $2005\left(r=-0.88, r^{2}=0.77\right)$.

Al comparar los periodos prevacunal y vacunal, entre 1990 y 1997 se registraron 391288 casos. En el periodo con programa indirecto de vacunación (19982000) se presentaron 84770 casos, lo que evitó 306 518 (disminución de 78.3\%). La puesta en marcha de

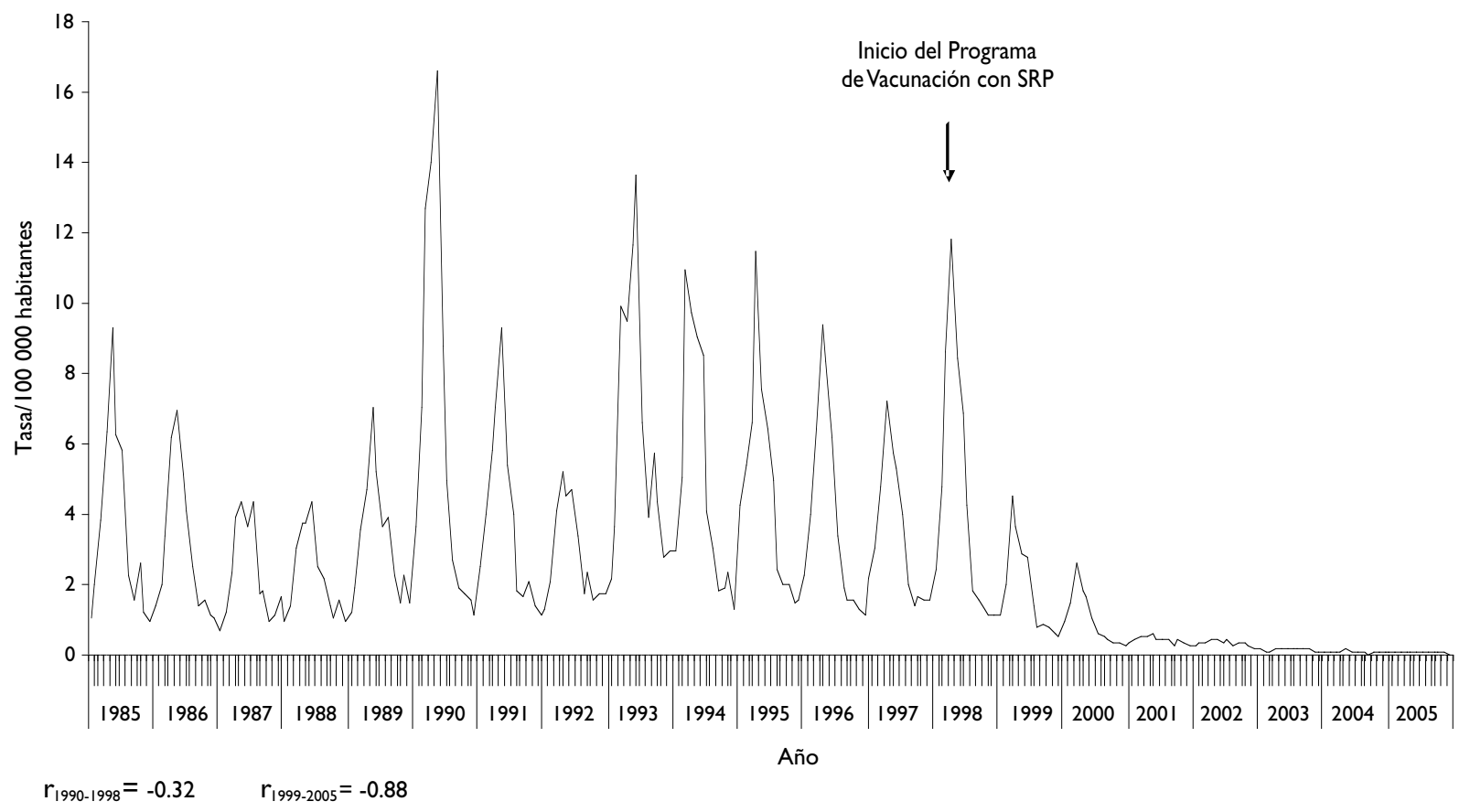

Fuente: Dirección General de Epidemiología. Anuarios de Morbilidad 1985-2005

Figura I. Incidencia de RUbeola en MéXico, 1985-2005 
la vacunación combinada (niños y adultos) posibilitó un ahorro adicional de 73454 casos de 2001 a 2005, lo que representa una disminución acumulada de $97.1 \%$ en relación con los casos de los últimos ocho años del periodo prevacunal.

Después de la epidemia de 1990 (65 591 casos notificados) ya referida se presentaron dos picos epidémicos en 1993 (67 879 casos notificados) y 1998 (51 846 casos notificados), con periodos interepidémicos asimétricos entre las exacerbaciones de 1990 y 1993 (dos años) y 1993 y 1998 (cinco años) (figura 2). Puede observarse que la curva de casos de 1993 ingresa al corredor endémico al año siguiente, se sitúa por tres años consecutivos en la zona de alarma e ingresa a la zona de seguridad hasta 1997. A la introducción de la vacunación en el año epidémico de 1998 le siguió una rápida declinación, con descenso directo de la curva a la zona de éxito del espacio fase en 1999, que prosigue hasta 2005.

La figura 3 describe la variación estacional de la rubeola y delinea patrones cíclicos con exacerbaciones persistentes en los meses de primavera. La vacunación masiva atenúa esta variación y se advierte que en 2005 los casos no mostraron un patrón estacional específico.
El análisis por entidad federativa reveló que los estados de Aguascalientes, Baja California, Coahuila, Nuevo León, Quintana Roo, Tamaulipas y Yucatán, además del Distrito Federal, han presentado tasas de incidencia superiores a la media nacional, tanto en la época pre como en la vacunal. Cabe destacar que 13 estados han presentado discontinuidades transitorias aparentes de la transmisión local a partir de 2002 (Aguascalientes, Colima, Chihuahua, Hidalgo, México, Morelos, Nayarit, Puebla, Quintana Roo, San Luis Potosí, Sonora, Tabasco y Zacatecas). Destaca en especial Colima, con tres años continuos sin transmisión (2002-2004) y reincidencia en 2005; la discontinuidad se ha observado también en 20042005 en Morelos, Quintana Roo y San Luis Potosí.

La figura 4A delinea un incremento de la proporción de casos en menores de cinco años a partir de 2000, año de inicio de las medidas de vacunación en adultos; en la figura $4 \mathrm{~B}$ se observa que el aumento ocurre a expensas de los menores de un año. La figura $4 \mathrm{C}$ describe una elevación proporcional de casos en el grupo de 15 a 24 y de 25 a 44 años (intervalos que contienen a las MEF) entre 1998 y 2000; esta tendencia se revierte en parte a partir de 2001. En la figura 5A y B se reconoce una mayor incidencia en la fase prevacunal en menores de cinco años y los subgrupos de 15 a 44 años.
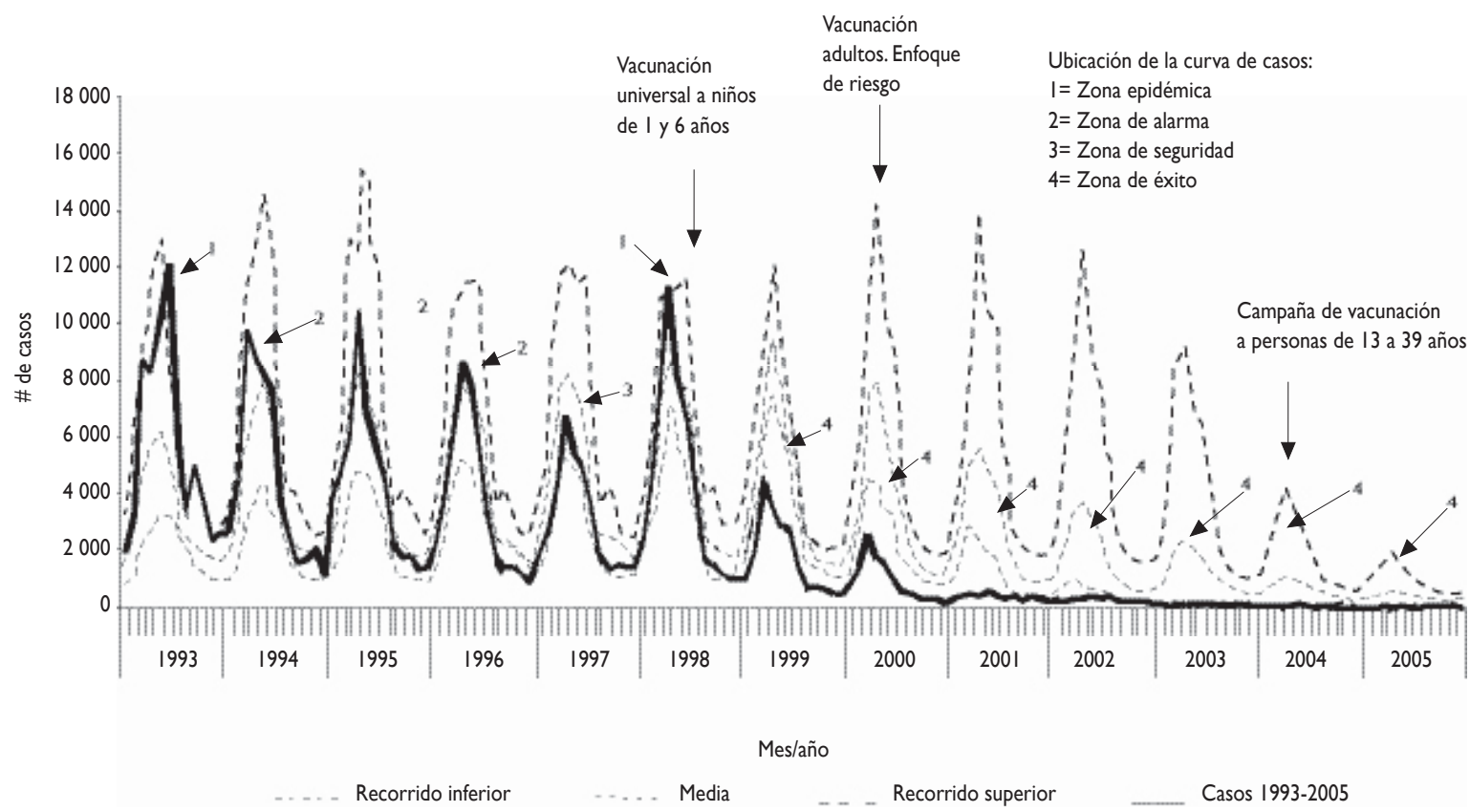

Fuente: Dirección General de Epidemiología. SSA

Figura 2. Corredor endémico de rubeola y Casos presentados en México, 1993-1995 


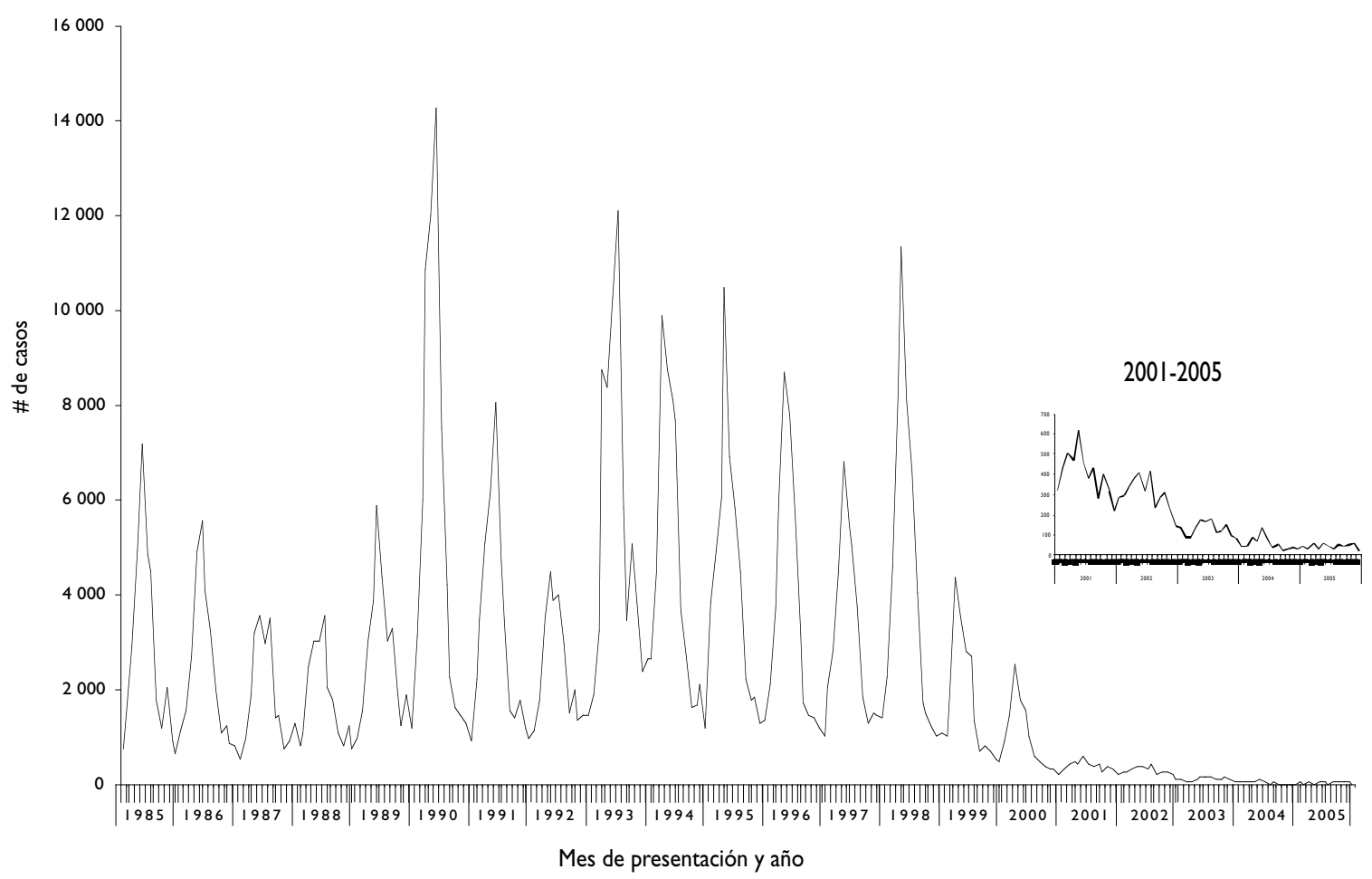

Fuente: Dirección General de Epidemiología. Anuarios de Morbilidad, 1985-2005

Figura 3.Variación estacional de casos de rubeola. México, 1985-2005

\section{Discusión}

La duración del periodo interepidémico entre 1993 y 1998 sugiere que en este lapso es posible una acumulación considerable de sujetos susceptibles; 7,22 en consecuencia, sin la vacunación quizá se habría presentado una epidemia de mayor magnitud y lenta declinación. Es notorio que la curva de casos de 1993 requirió cuatro años para alcanzar la zona de seguridad del corredor endémico; por el contrario, después de 1998 la curva se extendió rápidamente a la zona de éxito, cuya dinámica sugiere un aproximación a un punto de equilibrio estable próximo a cero casos, tal vez en relación con las coberturas de vacunación elevadas en preescolares y las medidas exitosas de vacunación suplementaria de 2004 y 2005 (vacunación indiscriminada a niños de 1 a 4 años y adultos de 13 a 39).9,16

La mayor frecuencia de casos en los meses de primavera del periodo prevacunal corresponde al patrón observado en enfermedades que se transmiten por vía respiratoria ${ }^{23}$ y se relaciona quizá con la acumulación de niños susceptibles en los salones de clase. Esta observación parece diluirse de 2004 a 2005 debido a la reducción del número de susceptibles en escolares, en virtud de la cobertura de vacunación elevada en preescolares y la aplicación de la segunda dosis a los seis años, en el mes de octubre, antes del inicio de la exacerbación de primavera. Estos resultados señalan la posibilidad de que se "horizontalice" la transmisión sin un patrón de estacionalidad claro, por lo que deben reforzarse las actividades permanentes de vacunación en unidades de salud a los grupos blanco del programa, incluidos los adultos hasta de 39 años sin antecedente documentado de las dosis requeridas para la edad.

Cabe señalar que algunos estados con tasas de incidencia superiores a la nacional se caracterizan por ejercer actividades comerciales o industriales importantes o experimentar un flujo turístico intenso, lo que incrementa la probabilidad de contacto entre sujetos susceptibles e infectados, condición necesaria para que se mantenga activa la cadena de transmisión. ${ }^{24}$ Esto debe alertar a los responsables de la vigilancia y prevención de la rubeola para orientar actividades preventivas con enfoque de riesgo. La heterogeneidad 


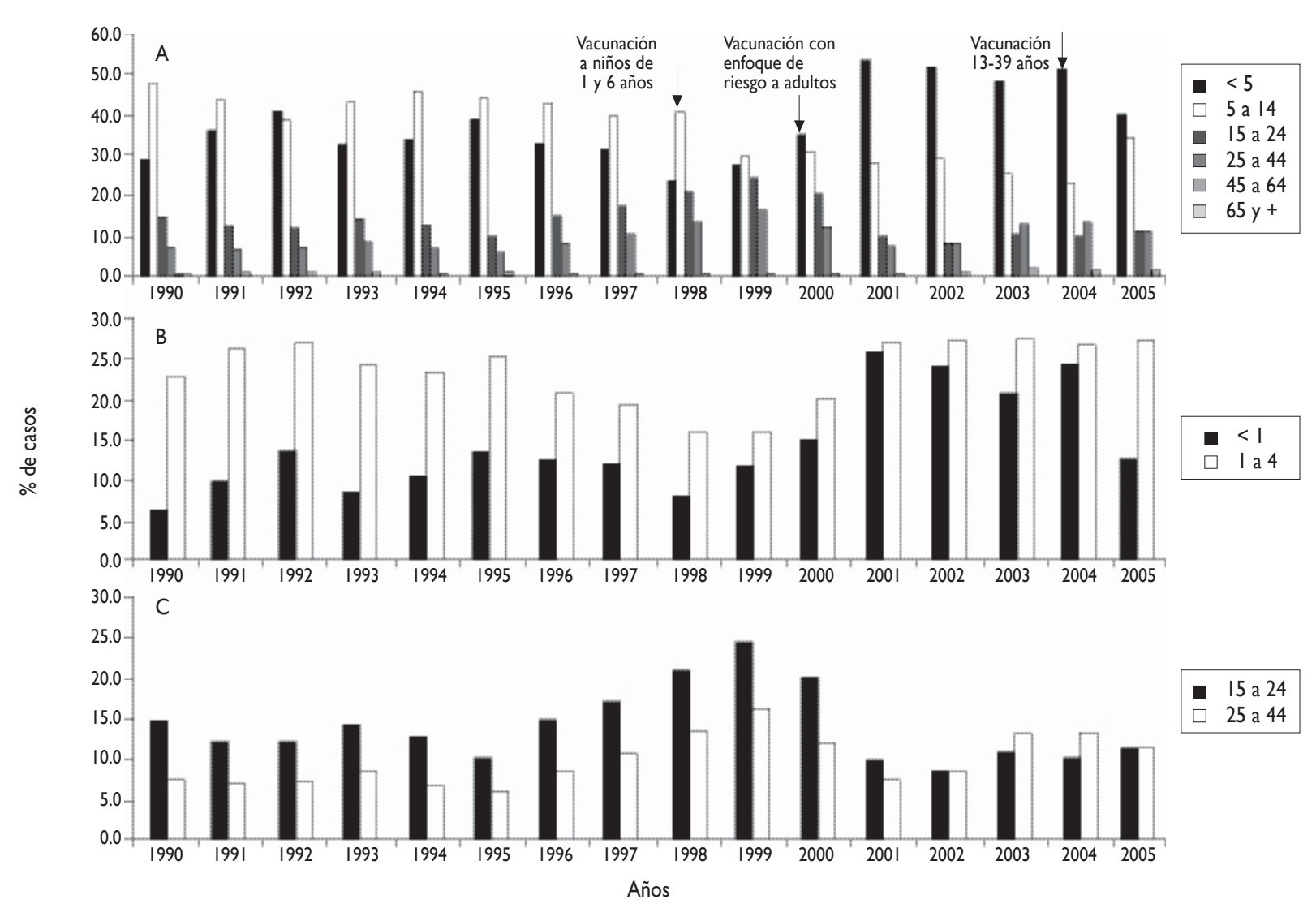

Fuente: Dirección General de Epidemiología.Anuarios de Morbillidad, 1985-2005

Figura 4. Proporción de casos de rubeola por Grupo de edad. México, 1990-2005
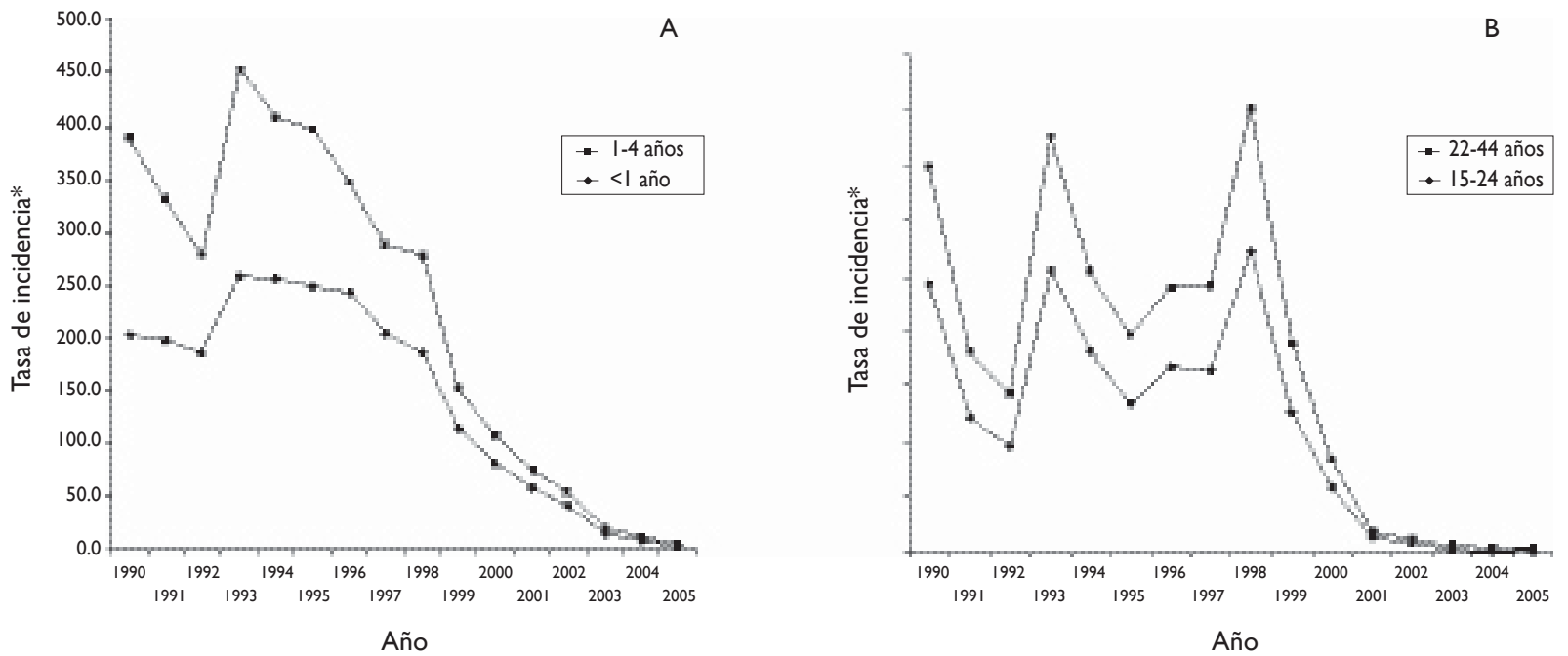

* Por 100000 habitantes de cada grupo de edad

Fuente: Dirección General de Epidemiología. Anuarios de Morbilidad, 1985-2005

Figura 5. Incidencia de rubeola en menores de 5 años y en Personas de I 5 a 44 años de edad. MéXico, 1990-2005 
de la distribución espacial de casos en años recientes es tal vez consecuencia de diferencias en la cobertura de vacunación oportuna y el reclutamiento de nuevos individuos susceptibles (migración de áreas rurales a ciudades), lo que se manifiesta por la interrupción transitoria de la transmisión en algunos estados y el descenso del número de casos en el país (537 casos notificados en 2005).

Como limitación, el estudio no posibilita hacer inferencias causales ${ }^{25}$ ni calcular el riesgo de infección, lo que es importante en la rubeola, ya que 30 a $50 \%$ de las infecciones puede ser subclínicas. ${ }^{1-2,26}$ Para la cuantificación del riesgo de infección son de utilidad las encuestas de seroprevalencia de anticuerpos; no obstante, el análisis de datos de morbilidad podría reflejar patrones de evolución de la infección ${ }^{27}$ que vale la pena puntualizar.

El programa de vacunación se inició con una medida indirecta de prevención del SRC, mediante la vacunación de niños a los 12 meses y seis años de edad; esto, de acuerdo con la epidemiología teórica, ${ }^{27,28}$ disminuye la fuerza de infección del padecimiento, definida como la tasa instantánea per cápita a la cual los sujetos susceptibles adquieren la infección ${ }^{28}$ y modifica el patrón de transmisión, con presentación de una mayor proporción de casos respecto de los de la fase prevacunal en individuos de 15 a 24 y 25 a 44 años. Este efecto de "rebote" se ha descrito como un patrón de transmisión esperado, atribuible a la vacunación indirecta, ${ }^{28}$ si bien a partir de 2001 se advierte un decremento irregular de la proporción de casos en adultos, con tasas de incidencia de 1999 a 2005 inferiores a las de años anteriores a la vacunación, caracterizados por cifras cercanas a $20 \%$ de susceptibilidad en MEF. 27,29 Los modelos teóricos predicen también el aumento de la proporción de casos en adultos debido a que la vacunación reduce la tasa reproductiva intrínseca de la enfermedad Ro, conocida como tasa de casos secundarios, lo que disminuye la oportunidad de contacto entre individuos susceptibles e infectados, prolonga los periodos interepidémicos y aumenta la edad promedio de infección. ${ }^{24,30-32}$

La introducción de las medidas de vacunación combinada en 2000 (niños y adultos) se acompañó de un incremento del número de casos en el grupo de menores de cinco años a expensas de los menores de un año. Tal desplazamiento, según los modelos teóricos desarrollados en Brasil, se vincula con la vacunación de individuos de edad superior a la promedio de infección, ${ }^{33,34}$ que en México era de 7 a 8 años de edad en el periodo prevacunal 1983 a $1990 .{ }^{35}$

Incluso cuando el modelo utilizado ${ }^{19}$ no permite realizar predicciones en el tiempo más allá del año siguiente al lapso analizado, cabe esperar que el des- censo de casos observado probablemente preceda a la interrupción nacional de la circulación endémica viral a mediano plazo, con un comportamiento de la rubeola exclusivamente epidémico, siempre que se mantengan las coberturas de vacunación oportuna elevadas y los programas de vacunación suplementaria exitosas. Este cambio debe reforzar los procedimientos de epidemiología molecular para identificar los genotipos virales y diferenciar casos relacionados con importación, de casos atribuibles a circulación autóctona viral.

Los cambios observados sugieren la conveniencia de reforzar la vigilancia de sarampión-rubeola, ${ }^{36-39}$ con base en investigación de casos, y no sólo de brotes para identificar factores vinculados con la transmisión, que orienten las actividades de vacunación suplementaria. Asimismo, debe aprovecharse la experiencia nacional e internacional en la definición de grupos con mayor riesgo de contraer y transmitir estos trastornos: trabajadores de la salud, magisterio, turismo y estancias infantiles, además de los trabajadores de plantaciones agrícolas e industrias maquiladoras en estados como Aguascalientes, Baja California, Tamaulipas, Sinaloa y Sonora, sitios que pueden atraer a individuos susceptibles provenientes de áreas rurales marginadas de otros estados del país. Hay que instituir formas de vigilancia del antecedente vacunal en el personal y estudiantes de nuevo ingreso en determinadas áreas profesionales (descritas con anterioridad) y educativas (normales, escuelas de medicina, enfermería, ciencias biológicas, laboratorio, turismo, etc.) y de abatimiento de oportunidades perdidas de vacunación en los grupos blanco del programa, además de reforzar la vacunación en mujeres en edad fértil no embarazadas y en el posparto inmediato.

\section{Referencias}

I. Miller E, Cradock-Watson JE, Pollock TM. Consequences of confirmed maternal rubella at successive stages of pregnancy. Lancet 1982;2:781-784. 2. Cutts FT, Robertson SE, Díaz-Ortega JL, Samuel R. Control of rubella and congenital rubella syndrome (CRS) in developing countries, part I: burden of disease from CRS. Bull World Health Organ 1997;75:55-68. 3. Robertson SE, Featherstone DA, Gacic-Dobo M, Hersh BS. Rubella and congenital rubella syndrome: global update. Pan Am J Public Health 2003; |4:306-315

4. Cutts F,Vynnycky E. Modelling the incidence of congenital rubella syndrome in developing countries. Int J Epidemiol 1999;28: I I76-I I84. 5. Orenstein WA, Bart KJ, Hinman AR, Preblud SR, Greaves WL, Doster SW, Stetler HC, Sirotkin B. The opportunity and obligation to eliminate rubella from the United States. JAMA 1984;25I:1988-1994.

6. Fine PM. Herd immunity: history, theory, practice. Epidemiol Rev 1993; 15:265-302.

7.Anderson RM, May RM. Infectious diseases of humans. Oxford: Oxford Science Publications; 1991:13-23. 
8. CDC.Achievements in public health: elimination of rubella and congenital rubella syndrome. United States, 1969-2004. MMWR 2005;54:279-282.

9. Dirección General de Epidemiología.Anuarios de morbilidad I9842006. Disponible en: http://www.dgepi.salud.gob.mx

10. Dirección General de Epidemiología. Manual para la vigilancia epidemiológica de sarampión. México: Secretaría de Salud; 1993.

II. Kim AJ, Roses PM. Elimination of rubella and congenital rubella sindrome in the Americas: another opportunity to address inequalities in health. Pan Am J Public Health 2004; I5: I45- 466

12. Eliminación de la rubeola y del síndrome de rubeola congénita. Guía práctica. Publicación científica y técnica 606. Washington: Organización Panamericana de la Salud; 2005.

13. Dirección General de Epidemiología. Procedimientos para la vigilancia epidemiológica de sarampión. Disponible en: http://www.salud.gob.mx. 14. Norma Oficial Mexicana NOM-036-SSA2-2002, para prevención y control de enfermedades, aplicación de vacunas, toxoides, sueros, antitoxinas e inmunoglobulinas en el humano. Diario Oficial de la Federación. México, DF, 21 de febrero de 200I.

15. Consejo Nacional de Vacunación. Manual de procedimientos técnicos de vacunación. Actualización 2003. México: Secretaría de Salud; 2003. 16. Disponible en: http://www.conava.gob.mx.

17. Aguirre Jaime A. Introducción al tratamiento de series temporales. Aplicación a las ciencias de la salud. Madrid: Editorial Díaz de Santos; 1994:25-I72.

18. Kleinbaum DG, Kupper DG, Morgenstern H. Epidemiologic research. Principles and quantitative methods. New York:Van Nostrand Reinhold Co.; 1982.

19. Bortman M. Elaboración de corredores o canales endémicos mediante planillas de cálculo. Rev Panam Salud Publica 1999;5: I-8.

20. Helfenstein U. Box-Jenkins modelling of some viral infectious diseases. Stat Med 1986;5:37-47.

2I. Thacker SB, Stroup DF. Public health surveillance. En: Browson RC,

Petitti DB, editors. Applied epidemiology. Oxford: Oxford University Press; 1998:105-135.

22. Fine PEM.The contribution of modeling to vaccination policy. En: Cutts FT, Smith PG, eds. Vaccination and world health. New York: John Willey \& Sons; 1994: I77-194.

23. Canals LM, Labra SF.Análisis no-lineal de la dinámica de enfermedades infecciosas en Chile. Rev Med Chil 1999;127:1086-1092.

24.Anderson RM, May RM. Directly transmitted infectious diseases: control by vaccination. Science 1982;215:1053-1060.
25. Susser M.The logic in ecological: I. The logic of analysis. Am J Public Health 1994;84:825-829.

26. Lehane DE, Newberg NR, Beam JrWE. Evaluation of rubella herd immunity during an epidemic. JAMA 1970;213:2236-2239.

27. José MV, Olvera J. La seroepidemiología de la rubeola en México: Datos y teoría. Salud Publica Mex 1992;34:328-334.

28. Knox EG. Theoretical aspects of rubella vaccination strategies. Rev Infect Dis 1985;7(suppl I):S194-S197.

29. Gutiérrez G, Muñoz O, Tapia Conyer R, Bustamante Calvillo ME, Álvarez y Muñoz MT, Guiscafré Gallardo H, Magos C, Sepúlveda J. Seroepidemiología de la rubeola en mujeres mexicanas. Encuesta Nacional Probabilística. Salud Publica Mex 1990;32:623-631.

30. José MV. El periodo interepidémico en poblaciones estacionarias y no estacionarias. Ciencia 1996;47:380-390.

31. Edmunds WJ, van de Heijden OG, Eerola M, Gay NJ- Modelling rubella in Europe. Epidemiol Infect 2000;125:6 17-634.

32. Reef SE, Frey TK, Theall K,Abernathy E, Burnett CL, Icenogle J, McCauley MM,Wharton M.The changing epidemiology of rubella in the 1990s. On the verge of elimination and new challenges for control and prevention. JAMA 2002;287:464-472.

33. Massad E, Nascimento Burattini M,Azevedo Neto RS, Yang HM, Cotinho FAB, Zanetta DMT.A model-based design of a vaccination strategy against rubella in a non-immunized community of Sao Paulo State, Brazil. Epidemiol Infect 1994; I I 2:579-594.

34. Massad E, Azevedo-Neto RS, Burattini MN, Zanetta DMT, Coutinho $F A B$, Yang $H M$ et al.Assessing the efficacy of a mixed vaccination strategy against rubella in Sao Paulo, Brazil. Int J Epidemiol 1995;24:842-850.

35. José MV, Olvera J, Serrano O. Epidemiología de la rubeola en México. Salud Publica Mex 1992;34:318-237.

36. Irons B, Lewis M], Dahl-Regis M, Castillo-Solórzano C, De Quadros CA. Strategies to eradicate rubella in English-Speaking Caribbean. Am J Public Health 2000;90:1545-I549.

37. Castillo-Solórzano C, Carrasco P,Tambini G, Reef S, Brana M, De Quadros CA. New horizons in the control of rubella and prevention of congenital rubella sindrome in the Americas. J Infect Dis 2003; I87(supppl I):SI46-SI52.

38. Castillo-Solórzano C,Andrus JK. Rubella elimination and improving health care for women. Emerging Infect Dis 2004;10:2017-202I.

39. Irons B, Carrasco P, Morris-Glasgow V, Castillo-Solórzano C, De Quadros CA. Integrating measles and rubella surveillance:The experience in the Caribbean.J Infect Dis 2003; I87(suppl I):SI53-SI 57. 